\title{
Pressure-Induced Melting of Confined Ice
}

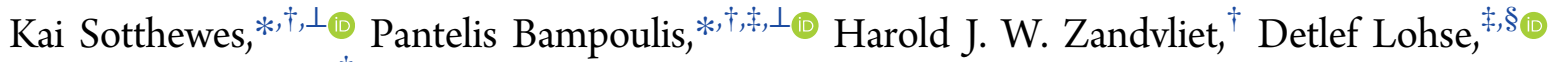
and Bene Poelsema

\begin{abstract}
${ }^{\dagger}$ Physics of Interfaces and Nanomaterials, Mesa+ Institute of Nanotechnology, and ${ }^{\ddagger}$ Physics of Fluids Group and Max Planck Center Twente, MESA+ Institute and J. M. Burgers Centre for Fluid Dynamics, University of Twente, P.O. Box 217, 7500AE Enschede, The Netherlands

${ }^{\S}$ Max Planck Institute for Dynamics and Self-Organization, Am Fassberg, 37077 Göttingen, Germany
\end{abstract}

\section{Supporting Information}

ABSTRACT: The classic regelation experiment of Thomson in the 1850s deals with cutting an ice cube, followed by refreezing. The cutting was attributed to pressure-induced melting but has been challenged continuously, and only lately consensus emerged by understanding that compression shortens the $\mathrm{O}: \mathrm{H}$ nonbond and lengthens the $\mathrm{H}-\mathrm{O}$ bond simultaneously. This $\mathrm{H}-\mathrm{O}$ elongation leads to energy loss and lowers the melting point. The hot debate survived well over 150 years, mainly due to a poorly defined heat exchange with
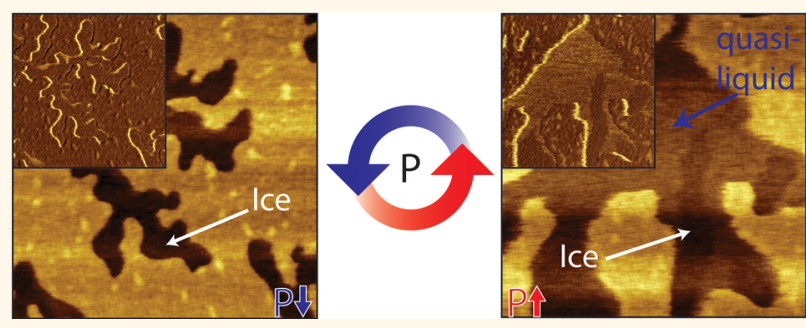
the environment in the experiment. In our current experiment, we achieved thermal isolation from the environment and studied the fully reversible ice-liquid water transition for water confined between graphene and muscovite mica. We observe a transition from two-dimensional (2D) ice into a quasi-liquid phase by applying a pressure exerted by an atomic force microscopy tip. At room temperature, the critical pressure amounts to about $6 \mathrm{GPa}$. The transition is completely reversible: refreezing occurs when the applied pressure is lifted. The critical pressure to melt the $2 \mathrm{D}$ ice decreases with temperature, and we measured the phase coexistence line between 293 and $333 \mathrm{~K}$. From a Clausius-Clapeyron analysis, we determine the latent heat of fusion of two-dimensional ice at $0.15 \mathrm{eV} / \mathrm{molecule}$, being twice as large as that of bulk ice. KEYWORDS: confined ice, pressure-induced melting, regelation, quasi-liquid layer, phase transition

W ater at atmospheric conditions exists in several states of aggregation, such as vapor, liquid, and several amorphous and crystalline solid phases. ${ }^{1-4}$ Understanding the vast amount of ice phases and phase transitions is essential for many fields, including environmental, life, and planetary sciences. ${ }^{5,6}$ The most important phase transitions are those of melting and freezing of water because they define the sea level and dominate life on Earth. ${ }^{7}$ One of the anomalous thermodynamic properties of water is that its melting point decreases as the pressure increases. ${ }^{8-11}$ This effect is of particular importance because it can define water flow under large compressive forces. Pressure-induced melting plays a prominent role in glacial motion. ${ }^{12-14}$ The weight of massive glaciers can cause internal deformations on the ice structure. The effect is strongest near the glacier/terrain interface, where pressures are highest. At these locations, ice melts even at temperatures below its bulk melting point, and the resulted liquid form of water allows the glacier to slide over the terrain.

It was initially believed that moderate pressures were sufficient to form a thin water layer on ice, attempting this way to explain the anomalous friction behavior of ice, for example, during ice skating. ${ }^{15}$ However, this idea was already challenged early on by Faraday. ${ }^{16}$ Slipperiness of ice (for example, in ice skating applications) is the result of the presence of a liquid-like film of water on the ice surface, even at temperatures below its freezing point. ${ }^{17-19}$ Pressure-induced melting of ice requires far greater pressures than those encountered in common slippery situations. Another example that is often associated with ice skating is Thomson's 19th century experiment that involves the sintering of a wire through an ice cube (or a large block of ice), ${ }^{16,20}$ the wire cuts through the ice by melting it by the application of an external force. As the wire moves through, the water behind it immediately refreezes. Ice melting due to the application of a high external pressure and refreezing when the pressure is relieved is known as regelation. Thomson's experiment is often used as a textbook paradigm for pressure-induced melting and regelation. ${ }^{21,22}$ However, even though pressure-induced melting is real when sufficiently high pressures are applied (in the order of hundreds of MPa or a few GPa), the wire that cuts through a block of ice is a far more complicated experiment and several other parameters contribute to the melting process. Among those, heat conduction through the wire, friction heating, and wire wettability contribute the most. ${ }^{11,23,24}$ Even though it is difficult to experimentally decouple pressure-induced melting from

Received: October 22, 2017

Accepted: November 7, 2017

Published: November 7, 2017 
other effects, it still plays a prominent role in several physical processes. It is most prominent in systems in which large pressures prevail. Such systems are difficult to access experimentally, and knowledge on the molecular dynamics comes only from theoretical investigations. ${ }^{11,25,26}$ It is thus highly desirable to find a way to access pressure-induced melting experimentally.

We have designed an experiment that allows for the first time to explore the microscopic behavior of ice layers under an external pressure. Our solution suppresses possible disturbing thermal influences from the environment. We use graphene as an ultrathin coating to trap water structures on a supporting mica surface. Because of graphene's unusual properties, such as impermeability to small molecules, mechanical flexibility, and chemical stability, it allows for the direct visualization of confined water structures by scanning probe techniques. ${ }^{27}$ The anisotropy in the thermal conductivity of graphene ${ }^{28}$ and mica, ${ }^{29}$ with a high/low conductivity parallel/perpendicular to the sheets, allows one to investigate the intrinsic properties of the ice network, isolated from thermal fluctuations during imaging. A sharp atomic force microscopy (AFM) tip is used to raster-scan the graphene surface on top of ice crystals on mica. By regulating the tip load, we can directly control the locally applied pressure at the graphene/ice/mica interface with nanometer precision and high accuracy. Any heat induced by the scanning AFM tip is quite rigorously led away from the ice crystals due to the extremely low thermal conductivity perpendicular to the graphene sheets as the in-plane thermal conductivity outweighs by far the out-of-plane thermal conductivity $\left(2000-4000\right.$ and $6 \mathrm{Wm}^{-1} \mathrm{~K}^{-1}$, respectively). ${ }^{28}$ The system is therefore a viable candidate to investigate pressure-related phase transitions of ice networks decoupled from thermal effects.

Graphene coating of water has provided useful insight on intercalation effects and on the physical properties of confined water structures. ${ }^{27,30-41}$ In principle, when water is confined between two flat surfaces, its structure and dynamics depend heavily on the molecular structure of the confinement walls, the confinement dimensions, temperature, and pressure. ${ }^{42-48}$ Often, confined water structures display perpendicular order due to stratification effects at the vicinity of the surface. ${ }^{49-51}$ In particular, water confined between graphene and mica forms flat islands with faceted edges and well-defined thickness, close to the interlayer distance of $\mathrm{I}_{\mathrm{h}}$ ice. ${ }^{27}$ These water structures are in equilibrium with the environmental water pressure, and they communicate with the environment through defects located at the graphene/mica interface. ${ }^{33}$ At ambient relative humidity $(\sim 50 \%)$, the graphene/mica confinement contains a thin water film with a thickness that corresponds to two water layers. ${ }^{52,53}$ Interestingly, at low relative humidity $(<1 \%)$, ice crystals grow at the interface induced by the heat extracted from the system by the evaporation of water molecules from the intercalated water film. ${ }^{53}$ Because of diffusion and rotational limitations of the water molecules that want to incorporate into the ice crystal, the crystallites acquire a fractal shape (see Figure 1a). ${ }^{54}$ The mica is hydrophilic and defines the structure of the ice crystal, whereas the graphene is slightly hydrophobic and acts as a neutral confinement. First-principle molecular dynamics (MD) simulations revealed that the first water monolayer is a fully connected hydrogen bonded network epitaxially grown on mica. ${ }^{55,56}$ The first ice layer on mica (and in contrast to multilayer films) has no free $\mathrm{O}-\mathrm{H}$ bonds sticking out of its surface. $^{55,57,58}$ The ice layer possesses a net dipole moment

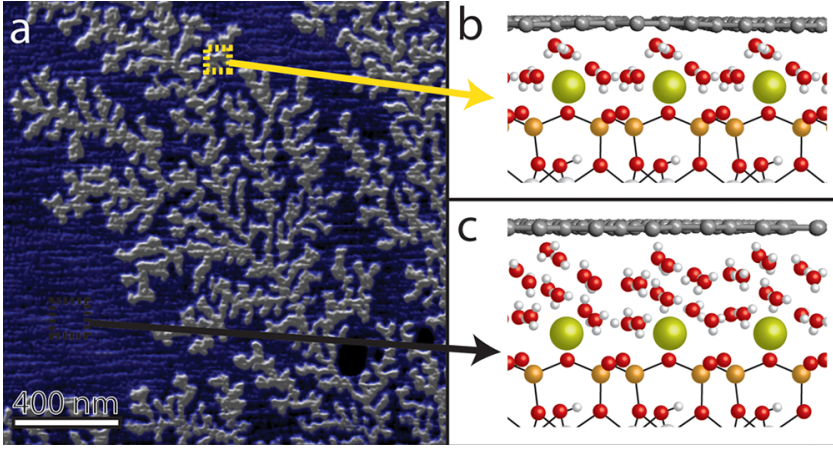

Figure 1. Structure of water intercalated between graphene and mica. (a) AFM topographic image $\left(1.5 \times 1.5 \mu \mathrm{m}^{2}\right)$ of ice crystals (bright region) confined between graphene and the supporting mica, surrounded by two water layers (dark region). (b,c) Schematic illustration of the structure of the ice crystals and the surrounding double layer of water, respectively. Color code: $\mathbf{O}$ (red), H (white), K (green), C (gray), Si (orange).

where the positive side points toward the mica surface. A schematic of this confined ice network is shown in Figure 1b, and the structure is based on ref 55. Owing to the absence of uncoordinated $\mathrm{O}-\mathrm{H}$ bonds on the surface of the ice layer and the appearance of a net dipole moment, a graphene covering these ice films is p-type doped. ${ }^{53,59}$

Here, we report on ice melting induced by the application of an external pressure. We show that the ice crystals melt when subjective to high external pressures and refreeze when the pressure is lifted, coined as regelation. For local pressures higher than $6 \mathrm{GPa}$, a solid to quasi-liquid transition takes place. The water molecules of the ice crystal become dynamic, and the layer loses its net dipole moment, indicative of disorder. The ice crystals start to melt initially at their edges, and the quasi-liquid layer expands toward their interior. The process is fully reversible when the applied pressure is released, and the water molecules immediately refreeze and reform a polarized ice layer. Our experiments are of interest to water (flow) in biological and geological systems. They also expand on the complex phase diagram of confined ice between graphene and mica.

\section{RESULTS AND DISCUSSION}

Pressure-Induced Solid to Quasi-liquid Phase Transition. When the graphene/water/mica system is exposed to low relative humidity, ice crystals are formed at the graphene/ mica interface induced by the heat extracted from the system due to water evaporation into the environment. ${ }^{53}$ An example is shown in the AFM topographic image shown in Figure 1a, where the ice crystals (shown as bright areas) have a fractal shape. The surrounding brighter area is a double layer water film (see Figure 1c); the height difference between the two levels in this image amounts to $0.36 \pm 0.02 \mathrm{~nm}$, a value very close to the interlayer distance of $I_{h}$ ice (hexagonal ice). The structure of the ice crystal is shown schematically in Figure $1 \mathrm{~b}$. Besides the ice crystals and the water bilayer, small droplets of water are occasionally present on top of the water double layer, as shown, for instance, in Figure 2a. The simultaneously recorded lateral force microscopy (LFM) image displays a difference in roughness between graphene on top of the ice layer and the surrounding water double layer. The higher roughness of graphene on top of the ice fractal was attributed to the presence of potassium ions and ionic domains on the air- 

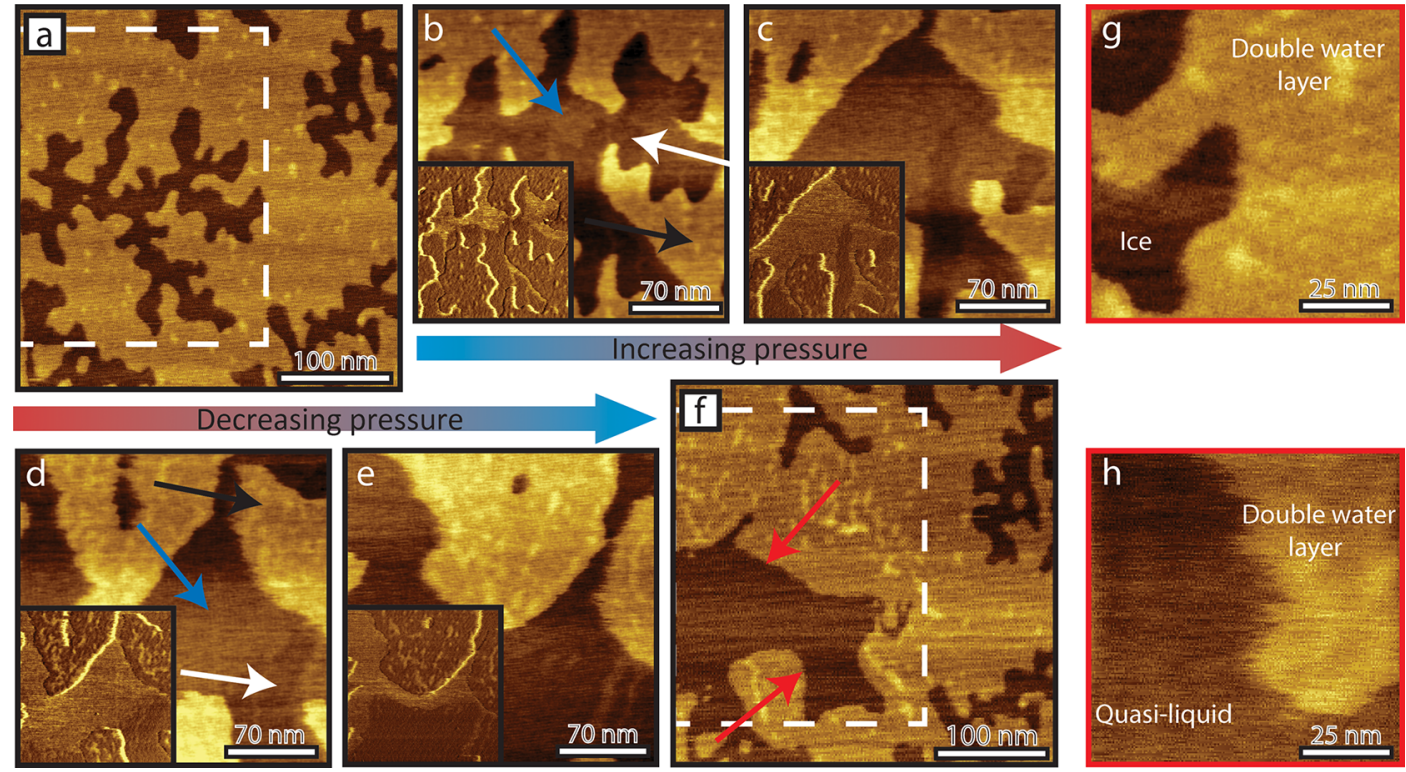

Figure 2. Behavior of confined water between graphene and mica under an external applied pressure. (a) AFM topographic image $(450 \times 450$ $\left.\mathrm{nm}^{2}\right)$ of an ice crystal (dark) measured with a tip load of $0.8 \mathrm{nN}(4.5 \mathrm{GPa})$. (b-e) Sequence of AFM topographic images $\left(230 \times 230 \mathrm{~nm}^{2}\right)$ and in the insets the corresponding LFM images showing the dynamic behavior of the ice fractals when there is pressure applied using an AFM tip. The applied pressures are approximately 8.5, 9.7, 10, and 8.5 GPa. Besides the ice layer (white arrow) and the double layer of water (black arrow), a third layer is present which grows with increasing pressure (blue arrow). This is the quasi-liquid water layer. (f) Same region as in (a), after several images were recorded with higher applied pressures within the white dashed box. The edges between the ice crystal and the double water layer become smooth after the pressure is lifted (red arrows). The fractal region increased and a higher density of water droplets is found on top of the double water layer. The area outside the white dashed borders is unaffected, indicating that the changes are only induced due to the pressure applied by the AFM tip. $(\mathrm{g}, \mathrm{h})$ Zoom-in topography images $\left(80 \times 80 \mathrm{~nm}^{2}\right)$ of a boundary between the ice crystal and the double layer of water at $4.5 \mathrm{GPa}(\mathrm{g})$ and of a boundary between the quasi-liquid layer and the double layer of water at $10 \mathrm{GPa}(\mathrm{g})$. A clear difference is observed in the fuzziness of the boundary.

cleaved mica surface (we note that the topography is featureless). ${ }^{59}$ The same structure is also present in the double water layer, however, less pronounced as a result of convolution by the second water layer. These images were obtained in contact mode AFM with a tip load of approximately $0.8 \mathrm{nN}$. By considering a p-doped diamond tip with a radius of curvature of about $5 \mathrm{~nm}$, the applied pressure on the graphene/ice/mica system induced by the tip is approximately $4.5 \mathrm{GPa}$, calculated using the Hertz model. ${ }^{60-62}$

Therefore, by scanning the surface in contact mode and varying the tip load in a controlled way, we can obtain spatial information about the aggregation state of the confined water structures as a function of the applied pressure. We find that when the pressure exceeds a critical value $\left(P_{c}\right)$, the ice/water edges become fuzzy and dynamic. These edges change from frame to frame even when the pressure is kept constant and higher than $P_{c}$. In addition, a quite faint but strongly persistent contrast appears that propagates from the edges of the ice crystal, visible in both topographic and LFM images (Figure $2 \mathrm{~b}, \mathrm{c}$ and their insets). The contrast is stronger in the LFM images. Under these conditions, the edges of this region are dynamic (see Figure $2 \mathrm{~h}$ and compare it to Figure $2 \mathrm{~g}$, i.e., a zoom-in of Figure 2a) and change shape every consecutive image, even when the pressure remains at a constant value. Furthermore, the area it occupies strongly depends on the applied pressure. As the pressure increases, this region propagates further toward the interior of the (dark) ice layer; this becomes clear upon comparing, for example, panels $\mathrm{c}$ and $\mathrm{d}$ of Figure 2, and the total area that it occupies increases further (see the movie in the Supporting Information for more details). The dynamic nature of this region suggests that the water molecules at this location are mobile. Based on this dynamic behavior, we will hereby refer to this region as a quasi-liquid water layer. Additional proof will be presented further below.

When the pressure is reduced, the area of the quasi-liquid layer shrinks, starting first from the interior of the ice crystal (Figure $2 \mathrm{~d}, \mathrm{e}$ ). The process is fully reversible, meaning that the molten area disappears completely when the pressure drops below a certain threshold $(<6 \mathrm{GPa})$. In addition to the disappearance of the quasi-liquid layer, the ice/double layer of water edges become stable and smooth when the pressure is decreased; see the red arrows in Figure 2f. Moreover, the density of small water droplets found on top of the surrounding water layers has increased, suggesting mass transport (see Figure $2 \mathrm{f}$ and compare it to $2 \mathrm{a}$ ). Note that after the pressure is lifted, the total ice area has increased, accompanied by a decrease of the double water layer area. The excess amount of water molecules form a third water "layer" or droplets on top of the double layer (mass conservation). Regions that were not scanned with a high tip load remained unaltered (see Figure 2a,f, outside the white dashed square). We emphasize that the melting of the ice crystals is heterogeneous, as it only occurs locally at the region of high pressure just below the surface of the AFM tip. As the tip moves across the graphene surface, the water is expected to refreeze at the locations left by the tip. At the locations where the pressure is lifted, refreezing should occur with a finite speed, that is, slower than the AFM scanning speed (the refreezing rate is low compared to the scan speed for a single line, $0.5 \mathrm{~s}$, but faster than the acquisition time of one image, $256 \mathrm{~s}$ ).

Figure $3 \mathrm{a}$ shows topographic information on an area consisting of the quasi-liquid layer, ice layer, and the double 


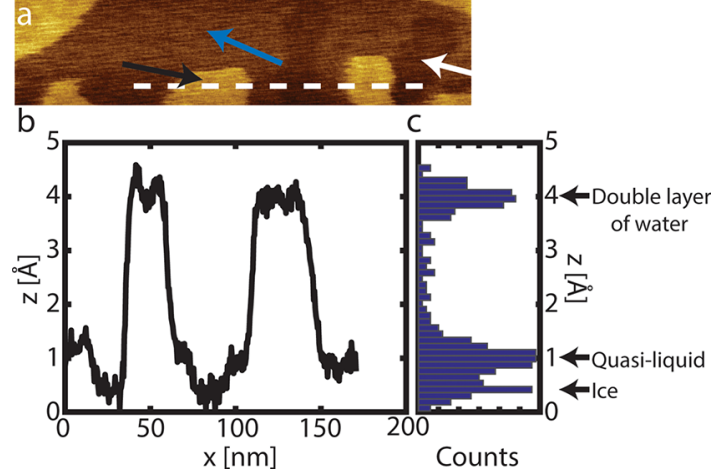

Figure 3. Height distribution of confined water under an external applied pressure larger than 6 GPa. (a) AFM topographic image $\left(230 \times 60 \mathrm{~nm}^{2}\right)$ of a melted fractal $(\sim 10 \mathrm{GPa})$. Three levels are present, namely, (i) the ice layer (indicated with a white arrow), (ii) the quasi-liquid layer (blue arrow), and (iii) the double water layer (black arrow). (b) Cross section across the white dashed line marked in panel (a). Three levels are present in the height profile. The height difference between the fractal and the double layer of water is approximately $3.6 \AA$. The quasi-liquid-like layer is approximately $70 \pm 5 \mathrm{pm}$ higher than the ice layer. (c) Histogram of the cross section in (b), showing three distinct peaks corresponding to the three different layers.

water layer. Marked with the white dashed line in Figure 3a, the line profile provides a quantitative measurement of the depth of the fractal with respect to the double layer of water, i.e., $0.36 \pm$ $0.02 \mathrm{~nm}$, which is in good agreement with previous studies. $^{34,52,53}$ A histogram of the line profile is shown in Figure $3 c$ and reveals a third peak which corresponds to the evolved quasi-liquid layer. This layer is approximately $70 \pm 5$ pm higher than the ice crystal. The increase of height is a result of the disordered water network, which is in direct contrast to the H-down network of the ice crystal (Figure 1b). The disorder results in several $\mathrm{OH}$ bonds that point away from the mica surface and increase the average thickness of the quasiliquid layer.

Supporting evidence that the evolved dynamic region is a quasi-liquid layer of water is obtained from LFM measurements (see insets in Figure $2 b-g$ ). Strikingly, the lateral deflection of the cantilever as measured by the LFM signal (which is proportional to tip-surface friction) increases at the regions where graphene covers the dynamic water layer by about $10 \%$. This is rationalized by the fact that in these regions it is easier to deform the graphene cover in a vertical sense (owing to the mobile nature of the water molecules at these locations). These indentations give then rise to enhanced resistance when the tip is moved parallel to the surface. On the other hand, graphene in contact with ice can be less easily indented, which gives rise to lower friction forces, in line with the observations. ${ }^{63,64} \mathrm{We}$ emphasize that the LFM images very clearly show the existence of melted ice and the extension of its area. These regions are also visible in topography images, but the contrast is rather weak and the exact area of the melt is sometimes harder to detect due to strong contrast enhancement actions.

Emerging Disorder in the Quasi-liquid Layer. In a previous study, we showed that the graphene cover can be doped by the underlying ice/water structures. ${ }^{59}$ That investigation made it possible to gain information about the structure of the ice crystal and the double water layer. The graphene on top of an ice crystal is p-doped, where on the other hand the double water layer does not induce any significant charge doping on the graphene cover because of disorder. The p-doping is the consequence of the crystalline structure of the ice, which has a $\mathrm{H}$-down configuration with a net dipole moment, ${ }^{55,56,58}$ whereas the net dipole moment is absent in the water double layer. In essence, the ice surface is electronegative, and the graphene is doped due to charge transfer. ${ }^{65}$ This difference in charge can be measured using a

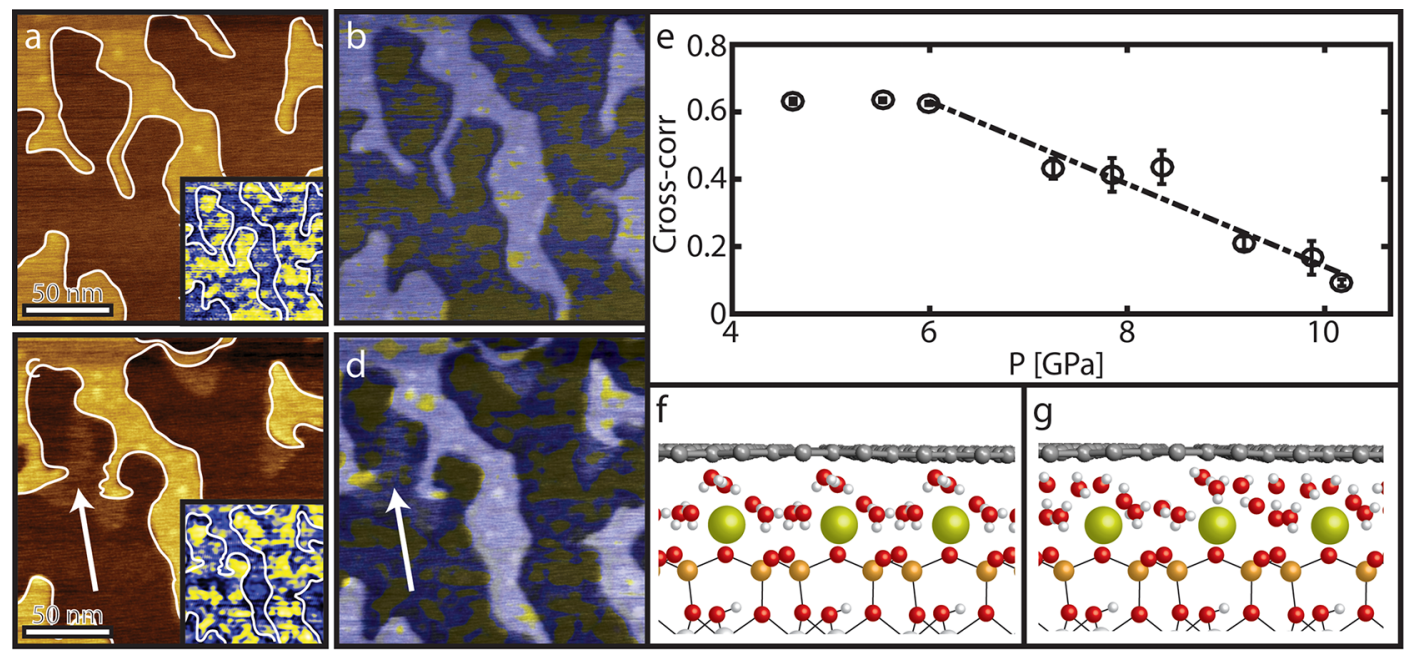

Figure 4. Graphene conductance and its relation to the water structure. (a) Topography image $\left(190 \times 190 \mathrm{~nm}^{2}\right) \mathrm{of}_{\text {an }}$ ice crystal intercalated between graphene and mica at room temperature when the tip load is $0.8 \mathrm{nN}$ (which corresponds to $4.5 \mathrm{GPa}$ ). As can be seen by the conductance image (inset), the charge (yellow) is localized at the graphene ice interface. (b) Corresponding overlaid images of the topography in (a) and its inset. A clear correlation is observed between the yellow conductance parts and the ice crystals. (c) Topography (inset: conductance) of an ice crystal scanned at an external pressure of $\sim 7 \mathrm{GPa}(3 \mathrm{nN})$. A quasi-liquid layer (indicated with a white arrow) is formed within the fractals borders. (d) Overlaid images of the topography in (c) and its inset. At the positions where the quasi-liquid layer is formed, the charge vanishes almost completely. (e) Cross-correlation between the topography and conductive images as a function of the applied pressure. A clearly diminishing trend is observed between the cross-correlation and the applied pressure. (f,g) Schematic illustrations of the ice layer and the quasi-liquid layer, respectively. Color code: $\mathrm{O}$ (red), $\mathrm{H}$ (white), $\mathrm{K}$ (green), $\mathrm{C}$ (gray), Si (orange). 
conductive AFM. ${ }^{59}$ Figure $4 \mathrm{a}$ and its inset show a topography and a conductive AFM image of an ice crystal intercalated between graphene and mica, under $4.5 \mathrm{GPa}$ of applied pressure (the pressure is small enough that it does not induce any changes in the ice crystal) at room temperature. No bias was applied between the conductive AFM tip and the substrate. Instead, only charges that are present on or near the surface can be detected by the AFM tip and measured in the current signal. A distinct correlation is found between the topography and the current image. The graphene layer on top of the ice layer displays a significant amount of current (yellow), whereas graphene above the double layer shows almost no current (blue). In order to enhance the correlation, the topography and the current images are overlaid in Figure $4 \mathrm{~b}$. Clearly, all the yellow parts (high current) are located within the borders of the ice fractal.

When pressures larger than $6 \mathrm{GPa}$ are applied on the ice crystals, a phase transition takes place and a quasi-liquid layer is formed (see Figure 4c: slightly brighter areas within the ice fractal). Exactly at the places where the quasi-liquid layer is formed, the current measured with the conductive AFM (CAFM) vanishes. When the two images are overlaid, the change in charge density becomes even more prominent (as shown in Figure $4 d$ ). This can only be explained by a change in the structure of the underlying ice crystal. As mentioned earlier, the water network in the ice has a H-down configuration, which results in a net dipole moment (Figure $4 \mathrm{f}$ ). ${ }^{55}$ The quasi-liquid layer, as a result of disorder, loses its net dipole moment, and therefore no current/charge is measured on the graphene cover (Figure $4 \mathrm{~g}){ }^{56}$ In Figure $4 \mathrm{e}$, the cross-correlation between the topography and the current image is shown. A distinct decrease of the correlation is observed as a function of the applied pressure. This declining in overlap is the expected result because, with increasing pressure, an increasing fraction of the ice layer is melted. As explained above, the melted regions do not contribute to the conduction.

To summarize the above observations, when the pressure exerted on the confined ice exceeds a specific threshold, the ice/water edges become very dynamic and a dynamic layer appears at the interface. This layer is thicker than the ice layer by $70 \pm 5 \mathrm{pm}$. This region increases in lateral size with increasing pressure. Owing to the dynamic nature of this layer, the observed disorder, and the high apparent mobility of the water molecules, we refer to it as a quasi-liquid water layer. We expect that this layer preserves some slight order that is stemming from the underlying mica due to stratification effects (see Figure $4 \mathrm{~g}$ ). We have thus shown that pressure variations can induce morphological changes in confined ice nanocrystals. The ice crystals melt when a high pressure is exerted at the interface by an AFM tip. When the pressure is lifted, the newly formed quasi-liquid layer refreezes. Our experiments provide the first ever example of regelation, fully decoupled from thermal effects owing to graphene's large anisotropy in the thermal conductivity, which warrants very good isolation form the environment. Heat that might be induced by frictional forces is immediately transported away from the underlying water structures, owing to the high in-plane thermal conductivity. This leaves pressure as the sole parameter responsible for the observed phase transitions.

The observed quasi-liquid layer shows similarities with the structure found by $\mathrm{Li}$ et al. on water on mica. ${ }^{56}$ The authors performed $a b$ initio molecular dynamics study of the structural and dynamic properties of water adlayers on the mica surface ${ }^{56}$ at different temperatures. They found that at room temperature molecules that are bonded to the mica form an ice network, where the water molecules bridging the $\mathrm{K}^{+}$ions are slightly weaker bonded than those bonded directly on the mica oxygen ions. When the system is brought to elevated temperatures, the structure starts to show melting behavior. Even though the hydrogen network collapses at these temperatures, the hydrogen bonds between the water and the supporting mica can remain. The bridging water molecules can easily rotate and diffuse, resulting in a liquid-like layer. Of course, in our system, the temperature remains constant and cannot be made accountable for the observed phase transition. We thus propose that the $\mathrm{H}_{2} \mathrm{O}$ molecules behave similarly when an external pressure is applied. When water is compressed, the $\mathrm{O}: \mathrm{H}$ hydrogen bond shortens and stiffens; on the other hand, the $\mathrm{O}-\mathrm{H}$ covalent bond elongates and softens via $\mathrm{O}-\mathrm{O}$ repulsion. ${ }^{26}$ The elongation of the covalent bond and its energy loss lowers the melting point. Once the pressure is reduced, the $\mathrm{H}: \mathrm{O}-\mathrm{H}$ bond fully recovers to its original state. ${ }^{25}$

Graphene Thickness Dependence. It is evident from the AFM images in Figure 2 that the quasi-liquid layer emerges at approximately $6 \mathrm{GPa}$, and its area increases in size when the external pressure is increased. The area of the quasi-liquid layer $\left(A_{\mathrm{QL}}\right)$ is measured for each frame and plotted as a function of the applied pressure in Figure 5a. The quasi-liquid layer area increases with increasing pressure until a maximum of $0.25 \mu \mathrm{m}^{2}$ ( $\sim 95 \%$ of the total ice area) at a pressure of approximately 10 $\mathrm{GPa}$. We note here that melting does not occur instantaneously everywhere in the image; these variations might originate from the non-homogeneous distribution of the potassium ions on the mica surface ${ }^{59}$ that could influence the bonding of the water network. ${ }^{56}$ When the pressure is decreased, the quasi-liquid area decreases until it completely vanishes. The molecules immediately refreeze and resume their positions in a polarized ice layer (see Figure 4a).

The same behavior is observed when the ice crystals are covered with thicker graphene covers. However, the applied force needed to create the quasi-liquid layer increases with the graphene thickness. For example, in order to melt an ice crystal covered by bilayer graphene, a $\sim 25 \%$ larger force is needed to melt the ice compared to monolayer graphene case (see Figure $5 b)$. For three layers of graphene, forces larger than $10 \mathrm{nN}$ are needed to form the quasi-liquid layer. We attribute this behavior to the increase of the effective tip-graphene contact area on the ice surface. Thicker graphene cover sheets will convolute the indentation by the tip more and lead to an increase of the effective contact area, due to their higher bending modulus ${ }^{66}$ compared to that of single-layer graphene. Therefore, higher forces are required in order to reach the pressure needed to melt the ice crystal (i.e., $6 \mathrm{GPa}$ ). The curves perfectly overlap with each other when compensating for the increase of the contact area due to the thicker graphene cover (inset of Figure $5 \mathrm{~b}$ ). This reveals that the mechanism leading to the observed phase transition is purely pressure. The extracted contact areas for bilayer and trilayer graphenes have increased about 2 and 3 times, respectively, compared to single-layer graphene.

Our information is obtained from friction forces during scanning, and the possibility that related heat effects may interfere with the inherent properties of the considered system needs attention. For this purpose, we have conducted experiments with different tips (different radii of curvature and material). The results of the most deviating measurements 
a

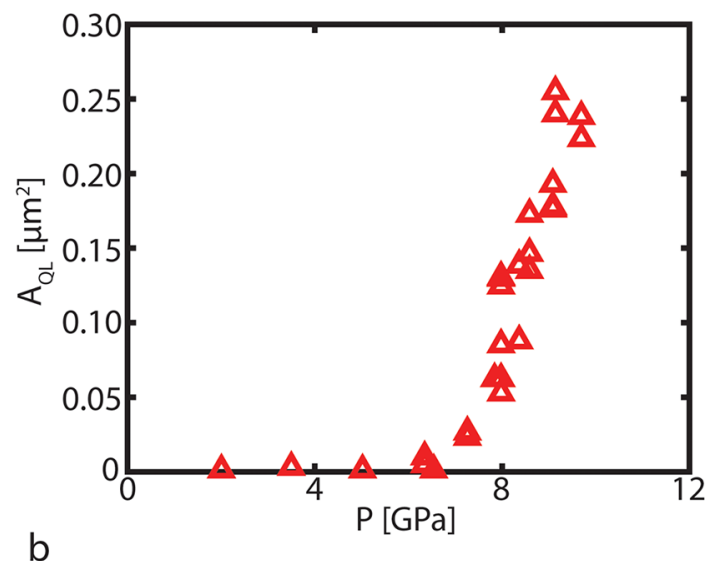

b

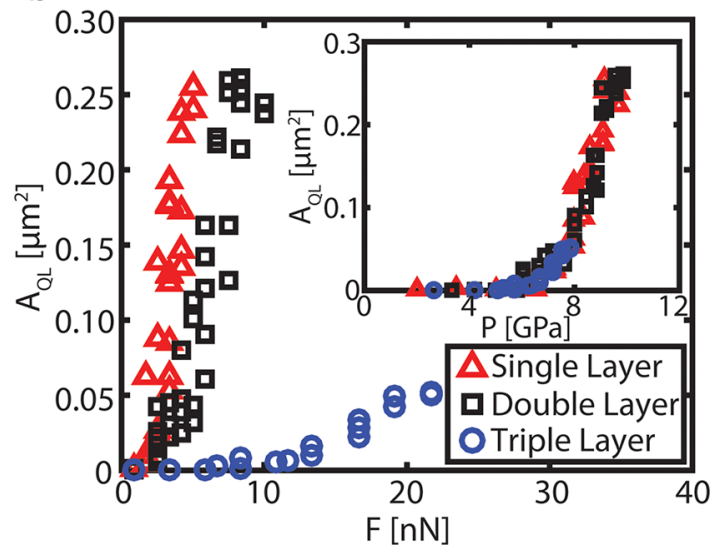

Figure 5. Influence of the graphene layer thickness on the melting behavior of confined ice. (a) Quasi-liquid layer area as a function of the applied pressure. The area of the quasi-liquid layer increases with increasing applied pressure and completely vanishes when the pressure is lifted. When the pressure is larger than $6 \mathrm{GPa}$, the ice crystals start to melt. (b) Area of the quasi-liquid layer as a function of the applied force for different graphene thicknesses recorded with the same diamond tip. Larger forces are needed to melt ice crystals when the number of graphene layers increases. Inset: Quasi-liquid layer area as a function of pressure for different number of graphene layers. Due to the increasing number of graphene layers, the effective contact area increases and thus a larger force is needed to melt the ice (while the required pressure remains constant).

are shown in Figure 5a, obtained with sharp diamond tip (radius of curvature $<5 \mathrm{~nm}$ ) and those shown in Figure 6a, obtained with a blunt Si tip (radius of curvature of $20 \mathrm{~nm}$ ), both from data gained at room temperature. When accounted for the different contact areas and the consequently larger forces required to melt the ice, the same melting characteristics are observed, and the influence of friction-induced heating on the melting of the ice crystals is thus clearly excluded. The clearest effect is expected from the variation of the contact area: a larger contact area leads to a higher friction force, ${ }^{67}$ and therefore, an enhanced heat generation should be expected. Still no differences are observed for the results obtained with tips with clearly different radii of curvature, and apparently, the graphene cover sheet warrants sufficient thermal insulation due to its anisotropic thermal conductivity discussed above. We can safely conclude that the melting of the ice is the result of the exerted pressure only.
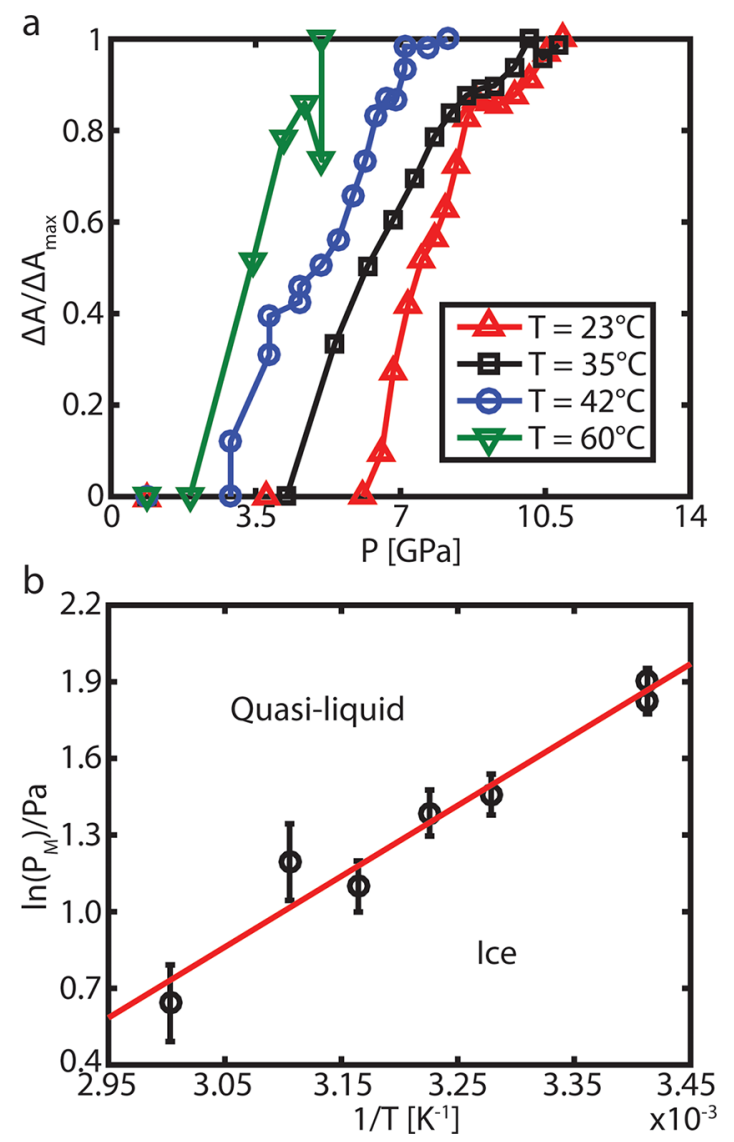

Figure 6. Phase diagram of confined water. (a) Areal change as a function of pressure at different substrate temperatures recorded with the same PtSi tip, normalized to the maximum quasi-liquid area. At elevated temperatures, the pressure needed to initiate melting decreases. (b) Phase diagram of confined water in the pressure-temperature $\left(\ln \left(P_{M}\right)\right.$ per $\left.\mathrm{Pa}, 1 / T_{M}\right)$ plane extracted from the curves in (a).

Temperature Influence on the Melting Pressure. When the temperature of the substrate is increased, the applied pressure required to melt the ice crystals decreases (see Figure $6 a)$. Because of the higher substrate temperature, the water molecules gain energy and therefore become dynamic even at lower pressures. ${ }^{56}$ As a result, less pressure is needed to melt the ice crystals. The magnitude depends strongly on the substrate temperature. For example, at $60{ }^{\circ} \mathrm{C}$, an external pressure of $\sim 2 \mathrm{GPa}$ is needed in order to melt the ice crystals. $\mathrm{Li}$ and $\mathrm{Zeng}^{56}$ predicted that for a monolayer of ice on mica without a graphene cover, the interfacial hydrogen bonds, that is, the bonds between the mica and the water molecules, are broken at temperatures around $100{ }^{\circ} \mathrm{C}$. At these temperatures, the ice layer loses its structure and its net dipole and acts as liquid. These observations explain the coarser and smoother shaped fractals observed after heating at $100{ }^{\circ} \mathrm{C}$ for $1 \mathrm{~h}$ in a recent study. ${ }^{53}$ When the temperature is increased, the fractals undergo edge melting, and the water molecules at the edges rearrange, resulting in a smoother and coarser fractal.

It is noted that the critical pressure for melting, $P_{\mathrm{M}}$, is equal to the summation of the van der Waals adhesion pressure, $P_{\mathrm{W}}$, and the critical exerted pressure, $P_{c}$. The van der Waals adhesion pressure is calculated using $P_{\mathrm{W}}=E_{\mathrm{W}} / d$, where $E_{\mathrm{W}}$ is the adhesion energy per unit area and $d$ is the distance between the graphene cover and the supporting mica. ${ }^{68,69} P_{\mathrm{W}}$ is 
estimated to be approximately $150 \mathrm{MPa}$ for $E_{\mathrm{W}} \approx 0.075 \mathrm{~J}$ $\mathrm{m}^{-236,54}$ and thus negligibly small. $P_{\mathrm{M}}$ as a function of temperature denotes the coexistence curve between the solid and the quasi-liquid phases. The functional shape of this curve is given by the Clausius-Clapeyron relation ${ }^{70}$

$$
\ln \left(\frac{P}{P_{0}}\right)=-\frac{L}{k}\left(\frac{1}{T}-\frac{1}{T_{0}}\right)
$$

where $L$ is the specific latent heat of fusion, $k$ is Boltzmann's constant, and $P_{0}$ is the equilibrium pressure at some temperature $T_{0}$. We have plotted the $\ln \left(P_{\mathrm{M}}\right)$ per $\mathrm{Pa}$ values obtained from data as shown in Figure $6 \mathrm{a}$ as a function of the corresponding reciprocal temperatures in Figure 6b. From a first-order polynomial fit and by using eq 1 , we have extracted the specific latent heat of fusion of water molecules from the quasi-liquid water into the solid ice and found it equal to $0.15 \pm$ $0.04 \mathrm{eV}$ per water molecule. We emphasize here that the value is independent of the uncertainty regarding the critical pressure of melting as the $\Delta A(P, T)$ curves have been obtained with the same tip. This value is only two times larger than the bulk latent heat of fusion at $0{ }^{\circ} \mathrm{C}$ (i.e., $0.062 \mathrm{eV}$ per water molecule). This correspondence is a strong confirmation of the suggested mechanism. The phase transition is clearly related to melting of the confined ice. The difference of the latent heat of fusion in two dimensions compared to the three-dimensional case is hard to explain and needs a specialized theoretical consideration, which is lacking at this moment. This difference is quite subtle in view of the fact that already in three dimensions the heat of fusion is about 1 order of magnitude smaller than the heat of vaporization. Interestingly, from our data, we can extrapolate that at about $100{ }^{\circ} \mathrm{C}$ the confined ice layer undergoes melting at a pressure of $\sim 1 \mathrm{~atm}$, in good agreement with the result of ref 53. We finally note that the exact value of $P_{\mathrm{M}}$ depends on the absolute size of the contact area. Possible margins have no consequence for the obtain heat of fusion of two-dimensional ice.

\section{CONCLUSIONS}

To conclude, the pressure-induced solid to quasi-liquid phase transition of confined ice has been explored in situ and real time using scanning probe microscopies. Two-dimensional ice crystals trapped between graphene and mica melt and form a quasi-liquid layer of water when a critical pressure beyond 6 $\mathrm{GPa}$ (at room temperature) is exerted locally onto the system. The H-down ice network loses its order, and the molecules become dynamic and mobile. The process is fully reversible; when the applied pressure is lifted, the water molecules immediately refreeze and resume a polarized $\mathrm{H}$-down network. We were able to determine the heat of fusion in $2 \mathrm{D}$ ice at 0.15 $\pm 0.04 \mathrm{eV}$ per water molecule. The protective graphene cover transports the dissipated energy induced by the probing tip effectively away from the ice crystals such that the melting and refreezing processes are only governed by pressure. The graphene cover warrants a powerful thermal protection from the environment. Our results are crucially important for understanding the phase behavior of confined water, and they provide an example of intrinsic regelation of $2 \mathrm{D}$ ice.

\section{EXPERIMENTAL SECTION}

The graphene flakes were obtained using the microexfoliation process from a freshly cleaved HOPG (ZYA grade, MikroMasch) and immediately deposited on a freshly cleaved mica surface (SPI, V1) at ambient conditions. The number of graphene layers was determined by optical microscopy with a DM2500H materials microscope (Leica, Germany) and tapping mode atomic force microscopy (Agilent 5100 atomic force microscope). ${ }^{53,71}$ All the experiments were performed inside an environmental chamber in which the relative humidity $(\mathrm{RH})$ can be controlled. The RH was measured using a humidity sensor (SENSIRION EK-H4 SHTXX, Humidity Sensors, Eval Kit, SENSIRION, Switzerland), with an accuracy of $1.8 \%$ between 10 and $90 \% \mathrm{RH}$ and was controlled by purging the environmental chamber with an adjustable $\mathrm{N}_{2}$ flow. The sample was heated using a Peltier element and a Lakeshore 332 temperature controller. Lateral force microscopy and conductive AFM imaging of the graphene-mica system was performed at room temperature and in contact mode using AD-E-0.5-SS tips (diamond tips, Adama Innovations) with a nominal spring constant of $0.3 \mathrm{~N} / \mathrm{m}$ and resonance frequency of $30 \mathrm{kHz}$ and PtSiCont (NanoSensors) with a nominal spring constant of $0.3 \mathrm{~N} / \mathrm{m}$ and a resonance frequency of $15 \mathrm{kHz}$. In order to make electrical contact with the graphene flakes, for the C-AFM measurements, the graphene flakes were mechanically connected with a bigger graphite flake acting as an electrode. ${ }^{59}$

\section{ASSOCIATED CONTENT}

\section{Supporting Information}

The Supporting Information is available free of charge on the ACS Publications website at DOI: 10.1021/acsnano.7b07472.

Movie of the topographic changes of confined water between graphene and mica when an external pressure is applied; when the pressure is increased, the quasi-liquid layer propagates from the edges of the ice crystal toward the interior; with a decreasing pressure, the quasi-liquid layer shrinks until it completely disappears when the pressure drops below the critical melting pressure (AVI) Movie of a sequence of lateral force images, recorded simultaneously with the topography movie, of confined water between graphene and mica when an external pressure is applied (AVI)

\section{AUTHOR INFORMATION}

\section{Corresponding Authors}

*E-mail: k.sotthewes@utwente.nl.

*E-mail: p.bampoulis@utwente.nl.

ORCID $\odot$

Kai Sotthewes: 0000-0003-2073-6958

Pantelis Bampoulis: 0000-0002-2347-5223

Detlef Lohse: 0000-0003-4138-2255

\section{Author Contributions}

${ }^{\perp}$ K.S. and P.B. contributed equally to this work.

\section{Notes}

The authors declare no competing financial interest.

\section{ACKNOWLEDGMENTS}

The authors thank the Dutch Organization for Research (NWO, STW 11431) for financial support.

\section{REFERENCES}

(1) Petrenko, V. F.; Whitworth, R. W. Physics of Ice; Oxford University Press, 1999.

(2) Malenkov, G. Liquid Water and Ices: Understanding the Structure and Physical Properties. J. Phys.: Condens. Matter 2009, 21, 283101.

(3) Libbrecht, K. The Physics of Snow Crystals. Rep. Prog. Phys. 2005, 68, 855-895. 
(4) Thomson, J. A Quantitative Investigation of Certain Relations Between the Gaseous, the Liquid and the Solid States of WaterSubstances. Proc. R. Soc. London 1873, 22, 27.

(5) Carr, M. H.; Head, J. W. Geologic History of Mars. Earth Planet. Sci. Lett. 2010, 294, 185-203.

(6) Thiel, P. A.; Madey, T. E. The interaction of Water With Solid Surfaces: Fundamental Aspects. Surf. Sci. Rep. 1987, 7, 211-385.

(7) Fairbanks, R. G. A. 17, 000-Year Glacio-Eustatic Sea Level Record: Influence of Glacial Melting Rates on the Younger Dryas Event and Deep-Ocean Circulation. Nature 1989, 342, 637-642.

(8) Schwegler, E.; Sharma, M.; Gygi, F.; Galli, G. Melting of Ice Under Pressure. Proc. Natl. Acad. Sci. U. S. A. 2008, 105, 1477914783.

(9) Tse, J.; Klug, D.; Tulk, C.; Swainson, I.; Svensson, E.; Loong, C.K.; Shpakov, V.; Belosludov, V.; Belosludov, R.; Kawazoe, Y. The Mechanisms for Pressure-Induced Amorphization of Ice Ih. Nature 1999, 400, 647-649.

(10) Tse, J. S.; Klein, M. L. Pressure-Induced Phase Transformations in Ice. Phys. Rev. Lett. 1987, 58, 1672.

(11) Hynninen, T.; Heinonen, V.; Dias, C. L.; Karttunen, M.; Foster, A. S.; Ala-Nissila, T. Cutting Ice: Nanowire Regelation. Phys. Rev. Lett. 2010, 105, 086102.

(12) Benn, D.; Evans, D. Glaciers and Glaciation; Routledge, 2014.

(13) Paterson, W. S. B. The Physics of Glaciers; Elsevier, 2016.

(14) Weertman, J. On the Sliding of Glaciers. J. Glaciol. 1957, 3, 3338

(15) Joly, J. The Phenomena of Skating and Professor J. Thomson's Thermodynamic Relation. Proc. R. Dub. Soc. 1887, 453-54.

(16) Faraday, M. Note on Regelation. Proc. R. Soc. London 1859, 10, 440.

(17) Rosenberg, R. Why is Ice Slippery? Phys. Today 2005, 58, 5054.

(18) Dash, J.; Rempel, A.; Wettlaufer, J. The Physics of Premelted Ice and Its Geophysical Consequences. Rev. Mod. Phys. 2006, 78, 695.

(19) Sánchez, M. A.; Kling, T.; Ishiyama, T.; van Zadel, M.-J.; Bisson, P. J.; Mezger, M.; Jochum, M. N.; Cyran, J. D.; Smit, W. J.; Bakker, H. J.; et al. Experimental and Theoretical Evidence for Bilayer-By-Bilayer Surface Melting of Crystalline Ice. Proc. Natl. Acad. Sci. U. S. A. 2017, $114,227-232$.

(20) Thomson, J. Note on Professor Faraday's Recent Experiments on Regelation. Proc. R. Soc. London 1860, 11, 198.

(21) Nye, J. Theory of Regelation. Philos. Mag. 1967, 16, 1249-1266.

(22) White, J. D. The Role of Surface Melting in Ice Skating. Phys.

Teach. 1992, 30, 495-497.

(23) Drake, L.; Shreve, R. Pressure Melting and Regelation of Ice by Round Wires. Proc. R. Soc. London, Ser. A 1973, 332, 51-83.

(24) Tozuka, S.; Wakahama, G. Studies on Regelation 1. Flow of Heat in the Regelation Process. J. Phys. Chem. 1983, 87, 4147.

(25) Zhang, X.; Huang, Y.; Sun, P.; Liu, X.; Ma, Z.; Zhou, Y.; Zhou, J.; Zheng, W.; Sun, C. Q. Ice Regelation: Hydrogen-Bond Extraordinary Recoverability and Water Quasisolid-Phase-Boundary Dispersivity. Sci. Rep. 2015, 5, 13655.

(26) Sun, C. Q.; Zhang, X.; Zheng, W. The Hidden Force Opposing Ice Compression. Chem. Sci. 2012, 3, 1455-1460.

(27) Xu, K.; Cao, P.; Heath, J. Graphene Visualizes the First Water Adlayers on Mica at Ambient Conditions. Science 2010, 329, 1188.

(28) Pop, E.; Varshney, V.; Roy, A. K. Thermal Properties of Graphene: Fundamentals and Applications. MRS Bull. 2012, 37, 1273.

(29) Gray, A.; Uher, C. Thermal Conductivity of Mica at Low Temperatures. J. Mater. Sci. 1977, 12, 959-965.

(30) Li, Q.; Song, J.; Besenbacher, F.; Dong, M. Two-Dimensional Material Confined Water. Acc. Chem. Res. 2015, 48, 119.

(31) Kim, J.-S.; Choi, J. S.; Lee, M. J.; Park, B. H.; Bukhvalov, D.; Son, Y.-W.; Yoon, D.; Cheong, H.; Yun, J.-N.; Jung, Y.; Park, J. Y.; Salmeron, M. Between Scylla and Charybdis: Hydrophobic GrapheneGuided Water Diffusion on Hydrophilic Substrates. Sci. Rep. 2013, 3, 2309.

(32) Ochedowski, O.; Bussmann, B.; Schleberger, M. Graphene on Mica-Intercalated Water Trapped for Life. Sci. Rep. 2015, 4, 6003.
(33) Severin, N.; Lange, P.; Sokolov, I. M.; Rabe, J. P. Reversible Dewetting of a Molecularly Thin Fluid Water Film in a Soft GrapheneMica Slit Pore. Nano Lett. 2012, 12, 774-779.

(34) Song, J.; Li, Q.; Wang, X.; Li, J.; Zhang, S.; Kjems, J.; Besenbacher, F.; Dong, M. Evidence of Stranski-Krastanov Growth at the Initial Stage of Atmospheric Water Condensation. Nat. Commun. 2014, 5, 4837.

(35) Wei, N.; Peng, X.; Xu, Z. Understanding Water Permeation in Graphene Oxide Membranes. ACS Appl. Mater. Interfaces 2014, 6, 5877-5883.

(36) Temmen, M.; Ochedowski, O.; Schleberger, M.; Reichling, M.; Bollmann, T. Hydration Layers Trapped Between Graphene and a Hydrophilic Substrate. New J. Phys. 2014, 16, 053039.

(37) Huang, H.; Song, Z.; Wei, N.; Shi, L.; Mao, Y.; Ying, Y.; Sun, L.; $\mathrm{Xu}, \mathrm{Z}$.; Peng, X. Ultrafast Viscous Water Flow Through NanostrandChannelled Graphene Oxide Membranes. Nat. Commun. 2013, 4, 2979.

(38) Wei, N.; Peng, X.; Xu, Z. Breakdown of Fast Water Transport in Graphene Oxides. Phys. Rev. E 2014, 89, 012113.

(39) Wu, K.; Chen, Z.; Li, J.; Li, X.; Xu, J.; Dong, X. Wettability Effect on Nanoconfined Water Flow. Proc. Natl. Acad. Sci. U. S. A. 2017, 114, 3358-3363.

(40) Dollekamp, E.; Bampoulis, P.; Faasen, D.; Zandvliet, H.; Kooij, E. Charge Induced Dynamics of Water in a Graphene-Mica Slit Pore. Langmuir 2017, 33, 11977-11985.

(41) Lee, H.; Ko, J.-H.; Choi, J.; Hwang, J.; Kim, Y.-H.; Salmeron, M.; Park, J. Enhancement of Friction by Water Intercalated between Graphene and Mica. J. Phys. Chem. Lett. 2017, 8, 3482-3487.

(42) Granick, S. Motions and Relaxations of Confined Liquids. Science 1991, 253, 1374-1379.

(43) Zhao, W. H.; Wang, L.; Bai, J.; Yuan, L. F.; Yang, J.; Zeng, X. C. Highly Confined Water: Two-Dimensional Ice, Amorphous Ice, and Clathrate Hydrates. Acc. Chem. Res. 2014, 47, 2505-2513.

(44) Bampoulis, P.; Teernstra, V. J.; Lohse, D.; Zandvliet, H. J.; Poelsema, B. Hydrophobic Ice Confined between Graphene and $\mathrm{MoS}_{2}$. J. Phys. Chem. C 2016, 120, 27079-27084.

(45) Cambré, S.; Schoeters, B.; Luyckx, S.; Goovaerts, E.; Wenseleers, W. Experimental Observation of Single-File Water Filling of Thin Single-Wall Carbon Nanotubes Down to Chiral Index $(5,3)$. Phys. Rev. Lett. 2010, 104, 207401.

(46) Koga, K.; Tanaka, H.; Zeng, X. C. First-Order Transition in Confined Water between High-Density Liquid and Low-Density Amorphous Phases. Nature 2000, 408, 564-567.

(47) Cicero, G.; Grossman, J. C.; Schwegler, E.; Gygi, F.; Galli, G. Water Confined in Nanotubes and Between Graphene Sheets: A First Principle Study. J. Am. Chem. Soc. 2008, 130, 1871-1878.

(48) Bampoulis, P.; Witteveen, J. P.; Kooij, E. S.; Lohse, D.; Poelsema, B.; Zandvliet, H. J. Structure and Dynamics of Confined Alcohol-Water Mixtures. ACS Nano 2016, 10, 6762-6768.

(49) Giovambattista, N.; Rossky, P. J.; Debenedetti, P. G. Phase Transitions Induced by Nanoconfinement in Liquid Water. Phys. Rev. Lett. 2009, 102, 050603.

(50) Koga, K.; Zeng, X. C.; Tanaka, H. Freezing of Confined Water: A Bilayer Ice Phase in Hydrophobic Nanopores. Phys. Rev. Lett. 1997, $79,5262-5265$

(51) Kimmel, G. A.; Matthiesen, J.; Baer, M.; Mundy, C. J.; Petrik, N. G.; Smith, R. S.; Dohnálek, Z.; Kay, B. D. No Confinement Needed: Observation of a Metastable Hydrophobic Wetting Two-Layer Ice on Graphene. J. Am. Chem. Soc. 2009, 131, 12838-12844.

(52) He, K. T.; Wood, J. D.; Doidge, G. P.; Pop, E.; Lyding, J. W. Scanning Tunneling Microscopy Study and Nanomanipulation of Graphene-Coated Water on Mica. Nano Lett. 2012, 12, 2665.

(53) Bampoulis, P.; Siekman, M. H.; Kooij, E. S.; Lohse, D.; Zandvliet, H.; Poelsema, B. Latent Heat Induced Rotation Limited Aggregation in 2D Ice Nanocrystals. J. Chem. Phys. 2015, 143, 034702.

(54) Bampoulis, P.; Lohse, D.; Zandvliet, H.; Poelsema, B. Coarsening Dynamics of Ice Crystals Intercalated Between Graphene and Supporting Mica. Appl. Phys. Lett. 2016, 108, 011601. 
(55) Odelius, M.; Bernasconi, M.; Parrinello, M. Two Dimensional Ice Adsorbed on Mica Surface. Phys. Rev. Lett. 1997, 78, 2855.

(56) Li, H.; Zeng, X. C. Two Dimensional Epitaxial Water Adlayer on Mica with Graphene Coating: An Ab Initio Molecular Dynamics Study. J. Chem. Theory Comput. 2012, 8, 3034.

(57) Verdaguer, A.; Sacha, G.; Bluhm, H.; Salmeron, M. Molecular Structure of Water at Interfaces: Wetting at the Nanometer Scale. Chem. Rev. 2006, 106, 1478-1510.

(58) Miranda, P.; Xu, L.; Shen, Y.; Salmeron, M. Icelike Water Monolayer Adsorbed on Mica at Room Temperature. Phys. Rev. Lett. 1998, 81, 5876.

(59) Bampoulis, P.; Sotthewes, K.; Siekman, M.; Zandvliet, H.; Poelsema, B. Graphene Visualizes the Ion Distribution on Air-Cleaved Mica. Sci. Rep. 2017, 7, 43451.

(60) Hertz, H. On the Contact of Rigid Elastic Solids. J. Reine Angew. Math. 1881, 92, 156.

(61) Bluhm, H.; Inoue, T.; Salmeron, M. Friction of Ice Measured Using Lateral Force Microscopy. Phys. Rev. B: Condens. Matter Mater. Phys. 2000, 61, 7760.

(62) Son, Y.; Wang, Q. H.; Paulson, J. A.; Shih, C.; Rajan, A. G.; Tvrdy, K.; Kim, S.; Alfeeli, B.; Braatz, R. D.; Strano, M. S. Layer Number Dependence of $\mathrm{MoS}_{2}$ Photoconductivity Using Photocurrent Spectral Atomic Force Microscopic Imaging. ACS Nano 2015, 9, 2843.

(63) Li, Q.; Lee, C.; Carpick, R. W.; Hone, J. Substrate Effect on Thickness-Dependent Friction on Graphene. Phys. Phys. Status Solidi B 2010, 247, 2909-2914.

(64) Lee, C.; Li, Q.; Kalb, W.; Liu, X.-Z.; Berger, H.; Carpick, R. W.; Hone, J. Frictional Characteristics of Atomically Thin Sheets. Science 2010, 328, 76-80.

(65) Rudenko, A.; Keil, F.; Katsnelson, M.; Lichtenstein, A. Graphene Adhesion on Mica: Role of Surface Morphology. Phys. Rev. B: Condens. Matter Mater. Phys. 2011, 83, 045409.

(66) Koskinen, P.; Kit, O. O. Approximate Modeling of Spherical Membranes. Phys. Rev. B: Condens. Matter Mater. Phys. 2010, 82, 235420.

(67) Mo, Y.; Turner, K. T.; Szlufarska, I. Friction Laws at the Nanoscale. Nature 2009, 457, 1116.

(68) Israelachvili, J. N. Intermolecular and Surface Forces; Academic Press, 2011.

(69) Algara-Siller, G.; Lehtinen, O.; Wang, F.; Nair, R.; Kaiser, U.; Wu, H.; Geim, A.; Grigorieva, I. Square Ice in Graphene Nanocapillaries. Nature 2015, 519, 443-445.

(70) Markov, I. V. Crystal Growth for Beginners: Fundamentals of Nucleation, Crystal Growth and Epitaxy; World Scientific, 2003.

(71) Dorn, M.; Lange, P.; Chekushin, A.; Severin, N.; Rabe, J. P. High Contrast Optical Detection of Single Graphenes on Optically Transparent Substrates. J. Appl. Phys. 2010, 108, 106101. 